\title{
Selbstbestimmtes Wohnen mit Unterstützung - Entwicklung und Überprüfung der „Modelltreueskala Selbstbestimmtes Wohnen (MSSW)“ für Menschen mit psychischen Beeinträchtigungen
}

\section{Supported Housing - Development and Validation of the “Supported Housing Fidelity Scale" for People With Mental Health Problems}

\section{다 (i) $\odot$}

\section{Autoren}

Dirk Richter ${ }^{1,2,3}$, Christine Adamus ${ }^{1,2}$, Sonja Mötteli ${ }^{4}$, Franziska Myszor ${ }^{5}$, Günther Wienberg ${ }^{6}$ Ingmar Steinhart $^{5}$

Institute

1 Zentrum Psychiatrische Rehabilitation, Universitäre Psychiatrische Dienste Bern, Schweiz

2 Klinik für Psychiatrie und Psychotherapie, Universität Bern, Schweiz

3 Departement Gesundheit, Berner Fachhochschule, Bern, Schweiz

4 Klinik für Psychiatrie, Psychotherapie und Psychosomatik, Psychiatrische Universitätsklinik Zürich, Schweiz

5 von Bodelschwinghsche Stiftungen Bethel, Bielefeld, Deutschland

6 von Bodelschwinghsche Stiftungen Bethel, Bethel.regional, Deutschland

Schlüsselwörter

Wohnen, psychiatrische Rehabilitation, Modelltreueskala

Keywords

housing, psychiatric rehabilitation, fidelity scale

Online-Publikation 28.10.2021

Bibliografie

Psychiat Prax 2022; 49: 103-106

DOI 10.1055/a-1509-4666

ISSN 0303-4259

(c) 2021. The Author(s).

This is an open access article published by Thieme under the terms of the Creative Commons Attribution-NonDerivative-NonCommercial License, permitting copying and reproduction so long as the original work is given appropriate credit. Contents may not be used for commercial purposes, or adapted, remixed, transformed or built upon. (https://creativecommons.org/licenses/by-nc-nd/4.0/)

Georg Thieme Verlag KG, Rüdigerstraße 14,

70469 Stuttgart, Germany
Korrespondenzadresse

Dr. Dirk Richter, Zentrum Psychiatrische Rehabilitation, Universitäre Psychiatrische Dienste Bern, Murtenstraße 46, 3008 Bern, Schweiz

dirk.richter@upd.unibe.ch

\section{ZUSAMMENFASSUNG}

Ziel der Studie In der psychiatrischen Versorgung fehlt bisher eine umfassende Beschreibung der Struktur- und Prozessmerkmale unterstützter Wohnformen im deutschsprachigen Raum. Die Entwicklung und Testung einer Modelltreueskala für selbstbestimmtes Wohnen wird dargestellt. Methodik Im Rahmen einer länderübergreifenden Kooperation wurde eine Skala entwickelt und in Interviews mit Leitungspersonen in 87 Diensten und Einrichtungen in Deutschland und der Schweiz eingesetzt.

Ergebnisse Die Modelltreueskala umfasst 31 Items in 4 Bereichen. Unterstützungsdienste in der eigenen Wohnung wiesen eine leicht höhere Modelltreue gegenüber besonderen Wohnformen (stationäre Einrichtungen) auf. Dies galt insbesondere für die Bereiche Wohnbedingungen und Inklusionsorientierung. In den Bereichen Mitarbeitende/ Team und Unterstützungsbedingungen wurden keine Unterschiede gemessen.

Schlussfolgerung Mit der Modelltreueskala steht erstmalig ein überprüfbarer Kriterienkatalog für das selbstbestimmte Wohnen in der psychiatrischen Versorgung zur Verfügung.

\section{ABSTRACT}

Objective For supported accommodation settings in mental health care, there is no comprehensive list of criteria for German-speaking countries available. The development and testing of a fidelity scale for independent housing is described.

Methods By means of a cross-country cooperation a fidelity scale was developed and was utilized in interviews with 
heads of 87 housing services and institutions in Germany and Switzerland.

Results The fidelity scale consists of 31 items in 4 topic fields. Outreach services demonstrated a slightly higher fidelity compared to residential care. This was particularly true for the topic fields 'Housing conditions' and 'Inclusion orientation'. We found not differences in the topic fields 'Staff/team' and 'Support conditions'.

Conclusions With this fidelity scale there is a testable list of criteria for independent housing services in mental health care available for the first time in German-speaking countries.

\section{Einleitung}

Die Situation des Wohnens für Menschen mit lang anhaltenden psychischen Erkrankungen hat sich in den vergangenen Jahrzehnten in den Ländern der westlichen Welt massiv gewandelt [1]. Nach der Deinstitutionalisierung der Versorgung und der Enthospitalisierung sogenannter Langzeitpatientinnen und -patienten ab den 1980er-jahren wurden zahlreiche Wohnheime, Wohngemeinschaften und betreute Wohnplätze errichtet. Ein abermaliger Wandel stellte sich ab dem Beginn der 2010er-Jahre ein, als das selbstbestimmte Wohnen in der eigenen Wohnung mit einer stundenweisen professionellen Unterstützung immer populärer wurde. Damit verbunden war ein Richtungswechsel in den Zielen der Wohnunterstützung, die sich - ebenso wie andere Bereiche der psychiatrischen Versorgung - nunmehr die Recovery- und Inklusionsorientierung zu eigen machte [2]. Gestützt wurde dieser abermalige Wandel vor allem in Deutschland durch veränderte gesetzliche Vorgaben und international durch große Studien im Bereich der Menschen mit psychischen Erkrankungen in Obdachlosigkeit, die ergeben haben, dass viele Betroffene selbst nach Jahren des Lebens auf der Straße in der Lage waren, in der eigenen Wohnung zurechtzukommen und ein stabiles Leben zu führen [3, 4].

Systematische Übersichtsarbeiten zu den Auswirkungen verschiedener Wohnformen, und hier insbesondere bezüglich des Vergleichs zwischen stationärem Wohnen und Unterstützung in der eigenen Wohnung, haben keine Nachteile für das Leben außerhalb von Einrichtungen gefunden [5-7]. Zudem besteht eine klare Präferenz der Nutzenden, die in einschlägigen Studien zu mehr als $80 \%$ den Wunsch äußerten, nicht in einem Heimsetting zu leben [8]. Diese Präferenz steht im Einklang mit der UN-Konvention für Rechte von Menschen mit Behinderungen, die in Artikel 19 zum Ausdruck bringt, dass Menschen mit Behinderungen das Recht haben sollen, ihren Wohnort frei zu wählen und nicht verpflichtet sind, in besonderen Wohnformen zu leben [9].

Das selbstbestimmte Wohnen mit Unterstützung hat viele Gemeinsamkeiten mit Wohnformen wie dem Ambulant Betreuten Wohnen in Deutschland oder mit dem Betreuten/Begleiteten Wohnen in der Schweiz. Allerdings kann ein wirklich selbstbestimmtes Wohnen in zentralen Merkmalen über diese Wohnformen hinausgehen. Bis anhin fehlen jedoch im deutschsprachigen Raum Kriterien, nach denen beurteilt werden kann, ob selbstbestimmtes Wohnen vorliegt. Dies ist insbesondere auch dann von Bedeutung, wenn Wohnsettings in ihrer Wirkung wissenschaftlich untersucht und verglichen werden. Hierzu braucht es zwingend transparente und messbare Kriterien [10]. Nachfolgend werden die Entwicklung und die Überprüfung einer Modelltreueskala beschrieben, welche diese Kriterien misst.

\section{Methode}

Modelltreueskalen haben sich in der psychiatrischen Rehabilitation vor allem im Bereich der Arbeitsrehabilitation für Supported-Employment-Programme durchgesetzt und als prädiktiv für den Programmerfolg herausgestellt $[11,12]$. Im Bereich des Wohnens sind bis anhin nur Instrumente in nordamerikanischen Studien bei Menschen in Obdachlosigkeit entwickelt worden (z. B. [13, 14]). Aufgrund der unterschiedlichen Sozialsysteme und der veränderten Anforderungen bei Menschen in Obdachlosigkeit, wurde auf eine Adaption der nordamerikanischen Skalen verzichtet und stattdessen unter Berücksichtigung der vorhandenen Instrumente eine Entwicklung für die hiesigen Anforderungen vorgenommen.

Im Rahmen einer länderübergreifenden Kooperation wurde die Skala im Rahmen zweier Forschungsprojekte entwickelt und getestet, die Wohnsettings in ihren Auswirkungen vergleichen. Dabei handelt es sich zum einen um eine Beobachtungsstudie, die von den v. Bodelschwinghschen Stiftungen Bethel/ Bielefeld durchgeführt wurde [15] und zum anderen um eine randomisierte kontrollierte sowie eine Beobachtungs-Interventions-Studie an den psychiatrischen Universitätskliniken in Zürich und Bern [16]. Beide Projekte untersuchen, ob das unterstützte Wohnen in der eigenen Wohnung oder in besonderen (stationären) Wohnformen zu unterschiedlichen Resultaten im Längsschnitt über einen Zeitraum von 24 Monaten führt. Beide Projekte werden mit denselben Erhebungsinstrumenten durchgeführt.

In einer multiprofessionellen Arbeitsgruppe wurde eine Modelltreueskala entwickelt. Sie basiert in Teilen auf relevanten Items der Fidelity-Skalen von Einrichtungen für Menschen in Obdachlosigkeit $[13,14]$ sowie auf einer britischen Arbeit über Merkmale von unterstützten Wohnsettings [17]. Nach der Neuformulierung der Merkmale für die Verhältnisse im deutschsprachigen Raum wurde die Skala unter Einbezug von Expertinnen und Experten mit Erfahrung sowie mittels einer Delphibefragung von professionellen Expertinnen und Experten zunächst einem Prätest und dann in einer Vollerhebung in persönlichen und telefonischen Interviews mit Leitungspersonen in Einrichtungen des Wohnens für Menschen mit psychischen Erkrankungen in Deutschland und in der Schweiz angewendet. Mittels eines Vergleichs von Unterstützungsdiensten in der eigenen Wohnung (nachfolgend UEW) mit besonderen Wohnformen, die eher dem stationären Wohnen zuzuordnen sind (nachfolgend BesWF), wurde die Skala auf diskriminative Validi- 
> Tab. 1 Kennwerte im Vergleich zwischen Unterstützungsangeboten in der eigenen Wohnung und besonderen Wohnformen.

\begin{tabular}{|c|c|c|c|}
\hline & $\begin{array}{l}\text { Unterstützungsangebote } \\
\text { in der eigenen Wohnung } \\
\mathrm{N}=54\end{array}$ & $\begin{array}{l}\text { besondere } \\
\text { Wohnformen } \\
\mathrm{N}=33\end{array}$ & $\begin{array}{l}\text { Wilcoxon-Rangsummen-Test** } \\
\text { (95\%-Konfidenzintervall) }\end{array}$ \\
\hline Gesamtskala (Mittelwert/Median) & $128,8 / 130$ & $124,6 / 125$ & $-4,99$ (-7,99 bis $-1,99)$ \\
\hline $\begin{array}{l}\text { Bereich Wohnbedingungen } \\
\text { (Mittelwert/Median } 6 \text { Items)* }\end{array}$ & $4,1 / 4,3$ & $3,5 / 3,5$ & $-0,66(-0,99$ bis $-0,50)$ \\
\hline $\begin{array}{l}\text { Bereich Mitarbeitende/Team } \\
\text { (Mittelwert/Median } 4 \text { Items) }^{*}\end{array}$ & $3,5 / 3,5$ & $3,7 / 3,5$ & $0,25(-0,00003-0,49)$ \\
\hline $\begin{array}{l}\text { Bereich Unterstützungsbedingungen } \\
\text { (Mittelwert/Median } 15 \text { Items)* }^{*}\end{array}$ & $4,2 / 4,2$ & $4,3 / 4,3$ & $0,06(-0,06-0,19)$ \\
\hline $\begin{array}{l}\text { Bereich Inklusionsorientierung } \\
\text { (Mittelwert/Median } 6 \text { Items) }^{*}\end{array}$ & $4,5 / 4,5$ & $4,2 / 4,2$ & $-0,33(-0,49$ bis $-0,00001)$ \\
\hline
\end{tabular}

tät geprüft. Hierzu wurden die Werte der Items in einer Summe aufaddiert. Die Datenerhebung erfolgte telefonisch. Es wurden Daten über 75 Settings in Deutschland und 12 Settings in der Schweiz erhoben. Von den 87 Settings entfielen 33 auf den BesWF-Bereich und 54 auf den UEW-Bereich. Die Durchführung der Studien wurde von den zuständigen Ethikkommissionen in der Schweiz (Swissethics, Nr. 2018-0238) und in Deutschland (Universität Münster, Nr. 2017-149-f-S) genehmigt.

\section{Ergebnisse}

Die neu entwickelte Modelltreueskala umfasst 31 Aussagen (s. online unter: http://osf.io/ztj6d/). Die Aussagen werden auf einer 5-stufigen Likertskala als Rating beantwortet ("trifft voll zu - trifft eher zu - teils/teils - trifft eher nicht zu - trifft gar nicht zu») und sind endpunktbenannt. Die Endpunkte verbalisieren ein Optimum und ein Pessimum. Beispielsweise lautet das Optimum (trifft zu) bei der Variable "Wahlfreiheit der Wohnform»: Dies bedeutet: alle Nutzenden haben volle Wahlfreiheit bei der Auswahl der Wohnform. Für das Pessimum (trifft gar nicht zu) lautet die Benennung der Ausprägung: Dies bedeutet: keine Nutzenden haben volle Wahlfreiheit bei der Auswahl der Wohnform. Maximal könnten auf der Summenskala 155 Punkte erzielt werden. Die Skala ist unterteilt in 4 theoretische Bereiche: Wohnbedingungen, Mitarbeitende/Team, Unterstützungsbedingungen, Inklusionsorientierung.

UEW-Angebote wiesen in der nicht normalverteilten Summenskala (Shapiro-Wilk-Test $w=0,9644 ; p=0,017$ ) insgesamt eine leicht bessere Modelltreue auf als BesWF-Angebote (Mittelwerte: UEW - 128,8 vs. BesWF - 124,6: Mediane: UEW 130 vs. BesWF - 125). Auf der Basis des $95 \%$-Konfidenzintervalls sind diese Unterschiede im Wilcoxon-Rangsummen-Test signifikant ( $\triangleright$ Tab. 1). Hinsichtlich der 4 oben genannten theoretischen Bereiche zeigte sich, dass UEW-Angebote bei den Wohnbedingungen und der Inklusionsorientierung eine signifikant höhere Modelltreue aufwiesen, nicht jedoch im Bereich Mitarbeitenden/Team sowie im Bereich der Unterstützungsbedingungen, wo sich keine Unterschiede ergaben. Zu beachten ist dabei, dass es sich bei den genannten Bereichen nicht um durch psychometrische Verfahren gebildete Subskalen handelt, sondern um fachlich-theoretische Zusammenhänge.

\section{Diskussion}

Die hier entwickelte Skala beschreibt erstmalig für den deutschsprachigen Raum Kriterien für eine Modelltreue für selbstbestimmtes Wohnen mit Unterstützung in Angeboten der psychosozialen Versorgung außerhalb der Einrichtungen für Menschen in Obdachlosigkeit. Aus methodischer Sicht haben Modelltreueskalen verschiedene Limitationen aufzuweisen. Da die Analyseeinheit ('unit of analysis') nicht eine Person, sondern ein Angebot psychiatrischer Dienstleistungen ist, fallen die Stichproben in der Regel recht klein aus, was die statistischen Möglichkeiten erheblich einschränkt.

Zudem sind gerade Fidelity-Skalen nicht notwendigerweise intern konsistent [12], was üblicherweise mit einem Maß wie Cronbach's Alpha demonstriert wird. Wenn, wie auch im Falle dieser Skala, zahlreiche Dienste einzelnen Kriterien nicht entsprechen, korrelieren die Items relativ schlecht. Während bei anderen Skalen dann in der Regel über den Ausschluss dieser Items nachgedacht wird, stellt sich diese Frage bei Modelltreueskalen eher nicht, da gerade diese Items aus fachlichinhaltlicher Sicht von Bedeutung sind. Eine weitere Limitation besteht darin, dass aus Ressourcengründen nur ein Interview pro Einrichtung oder Dienst geführt werden konnte, was eine Überprüfung der Interraterreliabilität nicht möglich machte.

Abweichend von anderen Modelltreueskalen in der psychiatrischen Rehabilitation wurden die Items endpunktbenannt, während in anderen Skalen oftmals jede Ausprägung beschriftet wird. Endpunktbenannte Fidelity-Skalen sind jedoch beispielsweise in der Überprüfung der Modelltreue bei psychotherapeutischen Verfahren nicht unüblich (s. etwa [18]).

Die Resultate hinsichtlich der Unterschiede zwischen Unterstützung in der eigenen Wohnung und besonderen Wohnformen fallen in etwa in der erwarteten Richtung aus. Selbstbestimmtes Wohnen mit Unterstützung erlaubt mehr Wahlfrei- 
heit und Inklusionsorientierung als ein Leben im stationären Setting. Dagegen stehen die Stabilität und Umfänglichkeit der Versorgung (etwa 24-Stunden-Erreichbarkeit) der stationären Einrichtungen, die viele aufsuchende Dienste nicht in dem gleichen Maße sicherstellen können. Deutlich wurde zudem, dass vor allem im Bereich Mitarbeitende/Team Verbesserungsbedarf unabhängig vom Wohnsetting besteht. Dies betrifft etwa die Mitarbeit von Expertinnen und Experten aus Erfahrung oder aber die Qualifikation von Mitarbeitenden hinsichtlich Gesprächsführung und Deeskalation. Es bestätigte sich auch in diesen Daten, dass nicht die Wohnform an sich den Unterschied macht, sondern bestimmte Qualitäten der Wohnunterstützung, hier etwa die Wahlfreiheit.

Die Modelltreueskala Selbstbestimmtes Wohnen bedarf zweifelsohne weiterer Anwendungen und Überprüfungen ihrer psychometrischen Eigenschaften. Die Skala, inklusive aller Materialien, steht der interessierten Öffentlichkeit frei zur Verfügung und kann über folgende Webseite heruntergeladen werden: http://osf.io/ztj6d/.

\section{Förderung}

Wir danken den teilnehmenden Diensten und Einrichtungen an den Studien in Deutschland und in der Schweiz. Die Datenerhebung erfolgte im Rahmen von zwei geförderten Projekten, in der Schweiz durch den Schweizerischen Nationalfonds zur Förderung der Wissenschaften (10531C_179451), in Deutschland durch die Förderung der Stiftung Wohlfahrtspflege NRW und der Stiftung Bethel.

Interessenkonflikt

Die Autorinnen/Autoren geben an, dass kein Interessenkonflikt besteht.

Literatur

[1] Richter D, Jäger M. Wohnforschung in der psychiatrischen Versorgung - Methodische Probleme und aktueller Forschungsstand. In: Konrad M, Rosemann M, Hrsg. Selbstbestimmtes Wohnen kompakt. Köln: Psychiatrie-Verlag; 2020: 92-102

[2] Farkas M, Coe S. From Residential Care to Supportive Housing for People With Psychiatric Disabilities: Past, Present, and Future. Front Psychiatry 2019; 10: 862. doi:10.3389/fpsyt.2019.00862

[3] Aubry T, Duhoux A, Klodawsky F et al. A Longitudinal Study of Predictors of Housing Stability, Housing Quality, and Mental Health Functioning Among Single Homeless Individuals Staying in Emergency Shelters. Am J Community Psychol 2016; 58: 123-135. doi:10.1002/ajcp.12067

[4] Aubry T, Goering P, Veldhuizen S et al. A Multiple-City RCT of Housing First With Assertive Community Treatment for Homeless Canadians
With Serious Mental Illness. Psychiatric services 2016; 67: 275-281. doi:10.1176/appi.ps.201400587

[5] Richter D, Hoffmann H. Independent housing and support for people with severe mental illness: systematic review. Acta Psychiatr Scand 2017; 136: 269-279. doi:10.1111/acps. 12765

[6] McPherson P, Krotofil J, Killaspy H. Mental health supported accommodation services: a systematic review of mental health and psychosocial outcomes. BMC Psychiatry 2018; 18: 128. doi:10.1186/s12888018-1725-8

[7] Gühne U, Stein J, Weinmann S et al. Wohninterventionen für Menschen mit schweren psychischen Störungen - Internationale Evidenz aus RCTs. Psychiat Prax 2017; 44: 194-205. doi:10.1055/s-0042118594

[8] Richter D, Hoffmann H. Preference for Independent Housing of Persons with Mental Disorders: Systematic Review and Meta-analysis. Administration and policy in mental health 2017; 44: 817-823. doi:10.1007/s10488-017-0791-4

[9] United Nations. UN Convention on the Rights of Persons with Disabilities. 2006: https://www.un.org/development/desa/disabilities/ convention-on-the-rights-of-persons-with-disabilities/conventionon-the-rights-of-persons-with-disabilities-2.html; Stand: 02.11.2020

[10] Gearing RE, El-Bassel N, Ghesquiere A et al. Major ingredients of fidelity: a review and scientific guide to improving quality of intervention research implementation. Clin Psychol Rev 2011; 31: 79-88. doi:10.1016/j.cpr.2010.09.007

[11] Bond GR, Peterson AE, Becker DR et al. Validation of the Revised Individual Placement and Support Fidelity Scale (IPS-25). Psychiatric services 2012; 63: 758-763. doi:10.1176/appi.ps.201100476

[12] Bond GR, Drake RE. Assessing the Fidelity of Evidence-Based Practices: History and Current Status of a Standardized Measurement Methodology. Adm Policy Ment Health 2020; 47: 874-884. doi:10.1007/s10488-019-00991-6

[13] Goering P, Veldhuizen S, Nelson GB et al. Further Validation of the Pathways Housing First Fidelity Scale. Psychiatric services 2016; 67: 111-114. doi:10.1176/appi.ps.201400359

[14] Stefancic A, Tsemberis S, Messeri P et al. The Pathways Housing First Fidelity Scale for Individuals With Psychiatric Disabilities. Am J Psychiatr Rehab 2013; 16: 240-261

[15] Projekt WieWohnen. Projekt WiEWohnen: Wirksamkeit der Eingliederungshilfe/Wohnen für Menschen mit seelischen Behinderungen. 2016: http://wiewohnen.de/; Stand: 21.10.2020

[16] Adamus C, Mötteli S, Jäger M et al. Independent Housing and Support for non-homeless individuals with severe mental illness: randomised controlled trial vs. observational study - study protocol. BMC Psychiatry 2020; 20: 319. doi:10.1186/s12888-020-02712-y

[17] McPherson P, Krotofil J, Killaspy H. What Works? Toward a New Classification System for Mental Health Supported Accommodation Services: The Simple Taxonomy for Supported Accommodation (STAX-SA). Int J Environ Res Public Health 2018; 15. doi:10.3390/ ijerph15020190

[18] Chawla N, Collin S, Bowen S et al. The mindfulness-based relapse prevention adherence and competence scale: development, interrater reliability, and validity. Psychother Res 2010; 20: 388-397. doi:10.1080/10503300903544257 